\title{
A Lie Algebraic Setting for Miura Maps Related to an Energy Dependent Linear Problem
}

\author{
Ian Marshall \\ School of Mathematics, University of Leeds, Leeds LS2 9JT, United Kingdom \\ Received February 13, 1990
}

\begin{abstract}
We study the affine semi-direct product, $\operatorname{Vect}\left(S^{1}\right) \ltimes C^{\infty}\left(S^{1}, \mathbb{R}\right) \otimes \mathbb{C}\left[\lambda, \lambda^{-1}\right]$ and show how the coadjoint action on its extended dual leads immediately to a generalisation of the Miura map and also to the existence of several compatible Hamiltonian structures.
\end{abstract}

\section{Introduction}

With a view to developing a clearer understanding of the underlying structures responsible for the exemplary behaviour of integrable systems, it is worthwhile trying to place results within the context of the now very familiar scenario of coadjoint actions of Lie groups. In this paper we are interested in looking at the so-called "energy-dependent" linear problem which is found to generate Miura maps (i.e. noninvertible Poisson maps) rather like the original one reiating the $\mathrm{MKdV}$ and $\mathrm{KdV}$ equations, see [3].

We follow the approach explained in a paper of Reyman and Semenov-TianShansky [1]. They showed how a family of $r$-brackets could be defined on an affine Lie algebra - these immediately giving rise to a family of compatible Poisson brackets on the dual. These authors further showed in [2] how this approach could be used to explain the existence of a family of Poisson structures associated with the problem

$$
\left(e \partial^{2}+u\right) \psi=0
$$

with $e$ and $u$ polynomials in the spectral parameter. Their analysis essentially amounts to the recognition that the relevant Lie algebra to look at is the Virasoro Lie algebra. In our case we consider an enlargement of the Virasoro algebra by means of the semidirect product.

Energy dependent Miura maps were investigated by a more direct method by Antonowicz and Fordy [3]. It should be emphasized that the structures described 
in [3] incorporate all those of the present paper. Our aim has been to find a Lie algebraic setting which can be said to explain them in terms of a coadjoint action.

To begin with we make some observations about coadjoint cocycles, see for example $[4,5]$. We then consider how to find coadjoint cocycles for - and so to extend - the semidirect product $\mathscr{L}=\mathfrak{g} \ltimes \mathscr{V}$ with $\mathfrak{g}=\operatorname{Vect}\left(S^{1}\right)$ and $\mathscr{V}=C^{\infty}\left(S^{1}, \mathbb{R}\right)$. The standard $\operatorname{ad}_{R}{ }^{*}$ Hamiltonian structures on the spaces $\hat{\mathscr{L}}^{*} \otimes \mathbb{C}\left[\lambda, \lambda^{-1}\right]$ and $\hat{\mathfrak{g}}^{*} \otimes \mathbb{C}\left[\lambda, \lambda^{-1}\right]$ are found to be related by a Miura map

$$
\hat{\mathscr{L}}^{*} \otimes \mathbb{C}\left[\lambda, \lambda^{-1}\right] \rightarrow \hat{\mathfrak{g}}^{*} \otimes \mathbb{C}\left[\lambda, \lambda^{-1}\right]
$$

Lastly we look at the connection with a spectral problem.

Remark. A nice explanation of the original Miura map comes from introducing the idea of a Poisson Group [6,7]. The mapping

$$
(\partial+v, \partial+w) \rightarrow(\partial+v)(\partial+w)
$$

is a Poisson mapping from $G \times G$ to $G$, where $G$ is the set of pseudo differential operators on the line/circle, and the Poisson structure on $G$ is the 2 nd Gelfand-Dikii bracket, or "Sklyanin bracket," given by

$$
\{f, g\}(L)=\operatorname{tr}\left[d g \cdot L \cdot(d f \cdot L)_{+}-L \cdot d g \cdot(L \cdot d f \mid)_{+}\right] .
$$

It can be shown that the map can be restricted to the subspace $\{(\partial+v, \partial-v)\}$, thus proving that $v \rightarrow\left(-v^{2}-v^{\prime}\right)$ is a Poisson map.

This construction cannot be reasonably extended to $G \otimes \mathbb{C}\left[\lambda, \lambda^{-1}\right]$.

\section{Preliminaries Regarding Cocycles}

Let $G$ be a Lie group and $\mathfrak{g}$ its Lie algebra, while $\mathfrak{g}^{*}$ is the dual space to $\mathfrak{g}$. A coadjoint group cocycle is a mapping $\Sigma: G \rightarrow \mathrm{g}^{*}$ satisfying

$$
\Sigma(g h)=\Sigma(g)+\operatorname{Ad}_{g}^{*} \Sigma(h) .
$$

The corresponding coadjoint Lie algebra cocycle $\sigma: \mathfrak{g} \rightarrow \mathfrak{g}^{*}$ must satisfy the infinitesimal version of this condition,

$$
d \Sigma(e)([X Y])=\sigma([X Y])=a d^{*}{ }_{X} \sigma(Y)-\operatorname{ad}^{*}{ }_{Y} \sigma(X) .
$$

We also find, by differentiating (1) with respect to $t$ at $t=0$ for $h=\exp (X t)$, that

$$
d \Sigma(g) \cdot d L_{g} \cdot X=\mathrm{Ad}_{g}^{*} \cdot d \Sigma(e) \cdot X
$$

Thus (3) is the equation we must solve in case we are given $\sigma=d \Sigma(e)$ and we wish to find $\Sigma$.

Suppose that $\sigma: \mathfrak{g} \rightarrow \mathfrak{g}^{*}$ and $\Sigma: G \rightarrow \mathfrak{g}^{*}$ are coadjoint cocycles with $\sigma=d \Sigma(e)$. Define $\omega: \mathfrak{g} \times \mathfrak{g} \rightarrow \mathbb{R}$ by

$$
\omega(X, Y)=\sigma(Y)(X) .
$$

If $\omega(X, Y)+\omega(Y, X)=0$, then we define the corresponding extension of $g$ to be the Lie algebra $\hat{\mathfrak{g}}=\mathfrak{g} \oplus \mathbb{R}$ with bracket,

$$
[(X, a),(Y, b)]=([X Y], \omega(X, Y)) \text {. }
$$


We easily find that the ad*-action of $\hat{\mathfrak{g}}$ on $\hat{\mathfrak{g}}^{*}=\mathfrak{g}^{*} \oplus \mathbb{R}$ is given by

$$
\operatorname{ad}_{(X, a)}^{*}(\ell, b)=\left(\operatorname{ad}^{*} \ell+b \sigma(X), 0\right) .
$$

As $a$ plays no rôle here we suppress it, and we regard (6) as defining the ad*-action of $\mathfrak{g}$ on $\hat{\mathrm{g}}^{*}$. It follows that the corresponding $\mathrm{Ad}^{*}$-action of $G$ on $\hat{\mathrm{g}}^{*}$ is given by

$$
\operatorname{Ad}_{g}^{*}(\ell, b)=\left(\operatorname{Ad}_{g}^{*} \ell+b \Sigma(g), b\right) \text {. }
$$

\section{The Semidirect Product $\mathscr{L}=\operatorname{Vect}\left(S^{1}\right) \ltimes C^{\infty}\left(S^{1}, \mathbb{R}\right)$}

Set $\mathrm{g}=\operatorname{Vect}\left(S^{1}\right)$ and $\mathscr{V}=C^{\infty}\left(S^{1}, \mathbb{R}\right)$. Then $\mathscr{L}$ is the semidirect product $\mathscr{L}=\mathfrak{g} \ltimes \mathscr{V}$ with Lie bracket given by

$$
[(\phi, v),(\psi, w)]=\left(\phi \psi^{\prime}-\phi^{\prime} \psi, \phi w^{\prime}-\psi v^{\prime}\right)
$$

Let us notice that $\mathscr{L}$ is isomorphic to the subalgebra $\{\phi \partial+v\}$ of the algebra of pseudo differential operators, and also - for future use - that $\mathscr{L}$ is isomorphic to the set of matrix differential operators

$$
\left\{\left(\begin{array}{cc}
-1 / 2 \phi^{\prime}+\phi \partial & 0 \\
-v^{\prime} & 1 / 2 \phi^{\prime}+\phi \partial
\end{array}\right)\right\}
$$

- in both cases with the ordinary commutation of operators as Lie bracket.

We will need the group $\mathscr{G}=G \ltimes \mathscr{V}$ corresponding to $\mathscr{L}$, given by $G=\operatorname{Diff}\left(S^{1}\right)=$ diffeomorphisms: $S^{1} \rightarrow S^{1}$, with the action of $G$ on $\mathscr{V}$ given by $g \cdot v=v \circ g$, where $\circ$ denotes composition of mappings. Thus the group multiplication in $\mathscr{G}$ is given by

$$
(g, v)(h, w)=(h \circ g, v+w \circ g) .
$$

Next we identify $\mathscr{L}^{*} \sim \mathscr{V} \times \mathscr{V}$ by means of the pairing $\langle\cdot, \cdot\rangle: \mathscr{L}^{*} \times \mathscr{L} \rightarrow \mathbb{R}$,

$$
\langle(u, \xi),(\phi, v)\rangle=\int u \phi+\int \xi v \text {. }
$$

We can derive all the various actions:

$$
\begin{aligned}
& \operatorname{ad}_{(\phi, v)}(u, \xi)=\left(2 \phi^{\prime} u+\phi u^{\prime}+v^{\prime} \xi,(\phi \xi)^{\prime}\right), \\
& \operatorname{Ad}_{(y, v)}(\phi, w)=\left(1 / g^{\prime} \phi \circ g, w \circ g-v^{\prime} / g^{\prime} \phi \circ g\right), \\
& \operatorname{Ad}_{(q, v)}(u, \xi)=\left(g^{\prime 2} u \circ g+v^{\prime} g^{\prime} \xi \circ g, g^{\prime} \xi \circ g\right) .
\end{aligned}
$$

One often finds in the literature the statement "Let us identify $g$ and $g^{*}$ by means of the (ad-invariant) inner product...", rather as in (11). It may be objected in the case $\operatorname{dim} \mathfrak{g}=\infty$ that this is not possible and that $\mathrm{g}^{*}$ is actually the much bigger space of distributions on $\mathfrak{g}$. The important thing to observe is that the coadjoint orbit in $\mathfrak{g}^{*}$ through any point $\alpha \in \mathrm{g}^{*}$ of the form $\alpha=\langle\hat{\alpha}, \cdot\rangle$ with $\hat{\alpha} \in \mathfrak{g}$ contains only points of the same form, and so we are effectively restricting our attention to reasonable Poisson subspaces.

We would like to find coadjoint cocycles for $\mathscr{L}$ and $\mathscr{G}$. It is known that the cohomology space $H^{2}(G, \mathbb{R})$ is one-dimensional, i.e. there is only one non-trivial coadjoint cocycle on $G$. We find that $H^{2}(\mathscr{G}, \mathbb{R})$ is three-dimensional. The following are coadjoint cocycles $\sigma_{i}: \mathscr{L} \rightarrow \mathscr{L}^{*}$, i.e. satisfy $\sigma_{i}([(\phi, v)(\psi, w)])=\operatorname{ad}_{(\phi, v)}^{*} \sigma_{i}(\psi, w)-$ 
$\operatorname{ad}_{(\psi, v)}^{*} \sigma_{i}(\phi, v):$

$$
\begin{aligned}
& \sigma_{1}(\phi, v)=\left(0, v^{\prime}\right), \\
& \sigma_{2}(\phi, v)=\left(v^{\prime \prime},-\phi^{\prime \prime}\right), \\
& \sigma_{3}(\phi, v)=\left(\phi^{\prime \prime \prime}, 0\right),
\end{aligned}
$$

(The cocycles $\sigma_{1}, \sigma_{2}$ also appear in a paper of Kupershmidt [8].)

We define the extended bracket on $\hat{\mathscr{L}}=\mathscr{L} \oplus \mathbb{R} \oplus \mathbb{R} \oplus \mathbb{R}$,

$$
\begin{aligned}
& {\left[\left(\phi, v, a_{1}, a_{2}, a_{3}\right)\left(\psi, w, b_{1}, b_{2}, b_{3}\right)\right]} \\
& \quad=\left(\phi \psi^{\prime}-\phi^{\prime} \psi, \phi w^{\prime}-\psi v^{\prime}, \int v w^{\prime}, \int\left(\phi^{\prime \prime} w-\psi^{\prime \prime} v\right), \int \phi \psi^{\prime \prime \prime}\right) .
\end{aligned}
$$

$\sigma_{3}$ gives rise to the Schwartzian derivative cocycle $\Sigma_{3}: \mathscr{G} \rightarrow \mathscr{L}^{*}$ as is well known. The other two give rise to new ones.

We want to find the group cocycles $\Sigma_{1}, \Sigma_{2}$ with the property $\sigma_{i}=d \Sigma_{i}(e)$. Using Eq. (3), we must solve:

i.e.

$$
d \Sigma_{i}(g, v) \cdot(\phi \circ g, w \circ g)=\operatorname{Ad}_{(g, v)}^{*} \sigma_{i}(\phi, w)
$$

$$
\left.\frac{d}{d t}\right|_{t=0} \Sigma_{i}(g+t \phi \circ g, v+t w \circ g)=\operatorname{Ad}_{(g, v)}^{*} \sigma_{i}(\phi, w) .
$$

We obtain

$$
\begin{aligned}
& \Sigma_{1}(g, v)=\left(\frac{1}{2} v^{\prime 2}, v^{\prime}\right), \\
& \Sigma_{2}(g, v)=\left(v^{\prime \prime}-v^{\prime} g^{\prime \prime} \mid g^{\prime},-g^{\prime \prime} / g^{\prime}\right), \\
& \Sigma_{3}(g, v)=(S(g), 0)=\left(\left(\frac{g^{\prime \prime}}{g^{\prime}}\right)^{\prime}-\frac{1}{2}\left(\frac{g^{\prime \prime}}{g^{\prime}}\right)^{2}, 0\right) .
\end{aligned}
$$

As in Eqs. (6) and (7) we extend the ad* and $\mathrm{Ad}^{*}$ actions to $\hat{\mathscr{L}}^{*}$, giving

$$
\begin{aligned}
\operatorname{ad}_{(\phi, v)}^{*}\left(u, \xi, e_{1}, e_{2}, e_{3}\right)= & \left(2 \phi^{\prime} u+\phi u^{\prime}+v^{\prime} \xi+e_{2} v^{\prime \prime}+e_{3} \phi^{\prime \prime \prime},(\phi \xi)^{\prime}+e_{1} v^{\prime}-e_{2} \phi^{\prime \prime}, 0,0,0\right), \\
\operatorname{Ad}_{(g, v)}^{*}\left(u, \xi, e_{1}, e_{2}, e_{3}\right)= & \left(g^{\prime 2} u \circ g+v^{\prime} g^{\prime} \xi \circ g+\frac{1}{2} e_{1} v^{2}+e_{2}\left(v^{\prime \prime}-v^{\prime} g^{\prime \prime} \mid g^{\prime}\right)+e_{3} S(g),\right. \\
& \left.g^{\prime} \xi \circ g+e_{1} v^{\prime}-e_{2} g^{\prime \prime} \mid g^{\prime}, e_{1}, e_{2}, e_{3}\right) .
\end{aligned}
$$

Now introduce a spectral parameter everywhere by setting

$$
\tilde{\mathscr{L}}=\hat{\mathscr{L}} \otimes \mathbb{C}\left[\lambda, \lambda^{-1}\right] \quad \text { and } \tilde{\mathscr{L}}^{*}=\hat{\mathscr{L}}^{*} \otimes \mathbb{C}\left[\lambda, \lambda^{-1}\right]
$$

and define the pairing $\tilde{\langle}, \tilde{\rangle}:(\mathscr{V} \times \mathscr{V}) \otimes \mathbb{C}\left[\lambda, \lambda^{-1}\right] \times \tilde{\mathscr{L}}$, in the usual way by $\tilde{\langle}(u, \xi),(\phi, v)\rangle=\left.\langle(u, \xi),(\phi, v)\rangle\right|_{\lambda-1}$; we drop the $\sim s$ on $\langle$,$\rangle from now on.$

Let $R_{k}=R \circ \overline{\lambda^{k}}$, where $R$ is the standard $r$-matrix $R=P_{+}-P_{-}$, where $P_{ \pm}$are the projections relative to the splitting $\mathbb{C}\left[\lambda, \lambda^{-1}\right]=\mathbb{C}[\lambda]+\mathbb{C}\left[\lambda^{-1}\right]$ and $\overline{\lambda^{k}}$ is the operator of multiplication by $\lambda^{k}$, i.e. $\bar{\lambda}^{k} \cdot X=\lambda^{k} X$. Then $\left\{R_{k}\right\}$ is a family of $r$-matrices on $\tilde{\mathscr{L}}$. Adjusting ad, a $\hat{\mathrm{d}}^{*}$ appropriately we get the Hamiltonian actions of $\tilde{\mathscr{L}}$ on $C^{\infty}\left(\tilde{\mathscr{L}}^{*}\right)$ defined by the a $\tilde{\mathrm{d}}^{*}{ }_{R_{k}}$-action for each $k$. These Hamiltonian actions are all compatible. This follows from the fact that $R(q)=R \circ \bar{q}$ is an $r$-matrix for any polynomial $q$ in $\lambda$, and is explained in detail in [1]. 
Integrable systems for the $\mathrm{a}^{*}{ }_{R_{k}}$-Hamiltonian systems are given by Hamiltonian functions which are invariants of the ordinary $\mathrm{A}^{*}$-action. Such functions then we need to find. To do this we simply observe that the combination

$$
w=e_{1} u-\frac{1}{2} \xi^{2}-e_{2} \xi^{\prime}
$$

is mapped by $\left(u, \xi, e_{1}, e_{2}, e_{3}\right) \rightarrow \operatorname{Ad}_{(y, v)}^{*}\left(u, \xi, e_{1}, e_{2}, e_{3}\right)$ to

$$
g^{\prime 2}\left(e_{1} u-\frac{1}{2} \xi^{2}-e_{2} \xi^{\prime}\right) \circ g+\left(e_{1} e_{3}+e_{2}{ }^{2}\right) S(g)=g^{\prime 2} w \circ g+\left(e_{1} e_{3}+e_{2}^{2}\right) S(g) .
$$

Hence invariants of the $A \tilde{d}^{*}$-action are eigenvalues of the monodromy matrix of the Sturm-Liouville equation,

$$
\left(2\left(e_{1} e_{3}+e_{2}^{2}\right) \partial^{2}+w\right) \Phi=0,
$$

with $w=e_{1} u-\frac{1}{2} \xi^{2}-e_{2} \xi^{\prime}$, cf. [2].

To find these invariants we look for an asymptotic series $\mathscr{A}=\Sigma A_{i} \lambda^{i}$ satisfying

$$
2\left(e_{1} e_{3}+e_{2}{ }^{2}\right) \mathscr{A}^{\prime}+2\left(e_{1} e_{3}+e_{2}^{2}\right) \mathscr{A}^{2}+w=0 \text {. }
$$

For some choices of $e_{1}, e_{2}, e_{3}$ (which are themselves elements of $\mathbb{C}\left[\lambda, \lambda^{-1}\right]$ ) this equation can be solved recursively, giving the integrable Hamiltonians

$$
f_{i}[w]=\int_{S^{1}} A_{i} .
$$

The family of functions found in this way is of course well known in the literature. Going back to (20) now we regard the mapping $(u, \xi) \rightarrow w$ as a generalised Miura map for which the functions of $w$ found above are pulled back to integrable Hamiltonian functions in $C^{\infty}\left(\tilde{\mathscr{L}}^{*}\right)$.

To be precise the mapping $m$, given by

$$
m\left(u, \xi, e_{1}, e_{2}, e_{3}\right)=\left(e_{1} u-\frac{1}{2} \xi^{2}-e_{2} \xi^{\prime}, e_{1} e_{2}+e_{2}{ }^{2}\right)
$$

is a Poisson mapping from $\tilde{\mathscr{L}}^{*}$ to $\tilde{\mathfrak{g}}^{*}$, where $\tilde{\mathfrak{g}}=\hat{\mathfrak{g}} \otimes \mathbb{C}\left[\lambda, \lambda^{-1}\right]$ and $\hat{\mathfrak{g}}=\mathfrak{g} \oplus \mathbb{R}$ is the extension of $\mathfrak{g}$ by means of the Schwartzian derivative cocycle. That is

$$
m^{*}\left(\{f, g\}_{\tilde{g}}\right)=\left\{m^{*} f, m^{*} g\right\}_{\tilde{\mathscr{L}}}
$$

for any $f, g \in C^{\infty}(\tilde{\mathfrak{g}})$.

In some cases the mapping will be invertible, but in others it will not be. Note:

In $[1]$ it is remarked that the subspace

$$
\ell_{-m n}=\left\{\sum_{-m}^{n} \lambda^{i} u_{i} \mid u_{i} \in \text { Lie algebra* }\right\}
$$

is a Poisson subspace for the Poisson bracket corresponding to $R_{k}=R \circ \overline{\lambda^{k}}$ if $-m \leqq k \leqq n+1$, and that if $-m \leqq k \leqq n, u_{n}$ can be fixed for all $t$. In our case inspection of (17) makes it clear that $\xi$ can be restricted further, for example:

$$
\begin{aligned}
& \left\{\left(\lambda^{-m} u_{-m}+\cdots+\lambda^{n} u_{n}, \lambda^{-s} \xi_{-s}+\cdots+\lambda^{r} \xi_{r}, \lambda^{-s} e_{1_{s}}+\cdots+\lambda^{r} e_{1_{r}}, \lambda^{-s} e_{2_{-s}}+\cdots\right.\right. \\
& \left.\left.\quad+\lambda^{r} e_{2_{r}}, \lambda^{-m} e_{3_{-m}}+\cdots+\lambda^{n} e_{3_{n}}\right) \mid-m \leqq-s \leqq r \leqq n\right\}
\end{aligned}
$$

is a Poisson subspace for the $k^{\text {th }}$ Poisson bracket provided that $-m \leqq-s \leqq$ $k \leqq r+1 \leqq n+1$; if $k \leqq n u_{n}$ can be fixed for all $t$, and if $k \leqq r \xi_{r}$ can be fixed for all $t$. 


\section{Examples}

\section{Example 1.}

$$
\begin{aligned}
& \quad e_{1}=-\lambda, \quad e_{2}=1, \quad e_{3}=0, \quad u=u_{0}=a \quad \text { say, } \xi=\xi_{0}=\eta \quad \text { say. } \\
& \text { (i.e. } \left.\frac{d}{d \lambda} u=0, \frac{d}{d \lambda} \xi=0\right) \\
& \mathrm{ad}^{*}{ }_{R_{k}\left(\lambda^{-k} \phi, \lambda^{\left.-k_{v}\right)}\right.}(a, \eta, \lambda, 1,0) \\
& =\lambda^{k}\left[\lambda^{-k}\left(2 \phi_{+}^{\prime} a+\phi_{+} a^{\prime}+v_{+}{ }^{\prime} \eta+v_{+}^{\prime \prime},\left(\phi_{+} \xi\right)^{\prime}-\lambda v_{+}^{\prime}-\phi_{+}^{\prime \prime}, 0,0,0\right)\right]_{-} \\
& -\lambda^{k}\left[\lambda^{-k}\left(2 \phi_{-}^{\prime} a+\phi_{-} a^{\prime}+v_{-}^{\prime} \eta+v_{-}^{\prime \prime},\left(\phi_{-} \xi\right)^{\prime}-\lambda v_{-}^{\prime}-\phi_{-}^{\prime \prime}, 0,0,0\right)\right]_{+} .
\end{aligned}
$$

If $k=0$, the right-hand side is

$$
\left(0, v_{1}{ }^{\prime}, 0,0,0\right) \text {, where } v_{-}=\lambda^{-1} v_{1}+\lambda^{-2} v_{2}+\cdots .
$$

That is we get the Hamiltonian structure given by

$$
\dot{\eta}=\left(\frac{\delta H}{\delta \eta}\right)^{\prime} \text {. }
$$

Also we can, for $k=0$, restrict $a=1$, as $a$ is a Casimir of the a $\widehat{d}^{*}{ }_{R_{k}}$-Hamiltonian structure. We compute

$$
\begin{aligned}
w & =e_{1} u-\frac{1}{2} \xi^{2}-e_{2} \xi^{\prime} \\
& =-\lambda-\frac{1}{2} \eta^{2}-\eta^{\prime}
\end{aligned}
$$

giving the original Miura map

$$
\eta \rightarrow-\frac{1}{2} \eta^{2}-\eta^{\prime}
$$

Example 2.

$$
\begin{aligned}
e_{1}=\lambda^{2}, \quad e_{2} & =1, \quad u=-\lambda^{2}+\lambda u_{1}+u_{0}, \quad \xi=\lambda \xi_{1}+\xi_{0}, \quad e_{3}=0 . \\
& =\lambda^{2} u-\frac{1}{2} \xi^{2}-\xi^{\prime} \\
& =-\lambda^{4}+\lambda^{3} u_{1}+\lambda^{2}\left(u_{1}-\frac{1}{2} \xi_{0}{ }^{2}\right)+\lambda\left(-\xi_{0} \xi_{1}-\xi_{0}{ }^{\prime}\right)+\left(-\frac{1}{2} \xi_{1}{ }^{2}-\xi_{1}{ }^{\prime}\right) \\
& =-\lambda^{4}+\lambda^{3} w_{3}+\lambda^{2} w_{2}+\lambda w_{1}+w_{0} \quad \text { say. }
\end{aligned}
$$

There are three compatible Poisson brackets for the "modified" $(u, \xi)$ side, corresponding to $k=0,1,2$ :

$$
\begin{aligned}
\{f, g\}_{k}= & \left\langle\left(-\lambda^{2}+\lambda u_{1}+u_{0}, \lambda \xi_{1}+\xi_{0}, \lambda^{2}, 1\right)\right. \\
& {\left[\left(\frac{\delta f}{\delta u_{0}} \lambda^{-1}+\frac{\delta f}{\delta u_{1}} \lambda^{-2}, \frac{\delta f}{\delta \xi_{0}} \lambda^{-1}+\frac{\delta f}{\delta \xi_{1}} \lambda^{-2}\right)\right.} \\
& \left.\left.\left(\frac{\delta g}{\delta u_{0}} \lambda^{-1}+\frac{\delta g}{\delta u_{1}} \lambda^{-2}, \frac{\delta g}{\delta \xi_{0}} \lambda^{-1}+\frac{\delta g}{\delta \xi_{1}} \lambda^{-2}\right)\right]_{R_{k}}\right\rangle .
\end{aligned}
$$

We can write down the Poisson structures - acting on $\left(\delta u_{0}, \delta \xi_{0}, \delta u_{1}, \delta \xi_{1}\right)$ as

$$
B_{0}=\left(\begin{array}{cc}
-J_{1} & -J_{2} \\
-J_{2} & 0
\end{array}\right), \quad B_{1}=\left(\begin{array}{cc}
J_{0} & 0 \\
0 & -J_{2}
\end{array}\right), \quad B_{2}=\left(\begin{array}{cc}
0 & J_{0} \\
J_{0} & J_{1}
\end{array}\right),
$$


with

$$
J_{0}=\left(\begin{array}{cc}
\partial u_{0}+u_{0} \partial & \xi_{0} \partial \\
\partial \xi_{0} & 0
\end{array}\right), \quad J_{1}=\left(\begin{array}{cc}
\partial u_{1}+u_{1} \partial & \xi_{1} \partial+\hat{\partial}^{2} \\
\partial \xi_{1}-\partial^{2} & 0
\end{array}\right), \quad J_{2}=\left(\begin{array}{cc}
-2 \partial & 0 \\
0 & \partial
\end{array}\right),
$$

so that

$$
\{f, g\}_{k}=\int\left(\frac{\delta f}{\delta u_{0}}, \frac{\delta f}{\delta \xi_{0}}, \frac{\delta f}{\delta u_{1}}, \frac{\delta f}{\delta \xi_{1}}\right) B_{k}\left(\begin{array}{c}
\delta g / \delta u_{0} \\
\delta g / \delta \xi_{0} \\
\delta g / \delta u_{1} \\
\delta g / \delta \xi_{1}
\end{array}\right) .
$$

There are five compatible Poisson brackets on the $w$ side corresponding to $k=0,1,2,3,4$ :

$$
\begin{aligned}
& \{F, G\}_{k}\left(-\lambda^{4}+\lambda^{3} w_{3}+\lambda^{2} w_{2}+\lambda w_{1}+w_{0}, 1\right) \\
& \quad=\left\langle\left(-\lambda^{4}+\lambda^{3} w_{3}+\lambda^{2} w_{2}+\lambda w_{1}+w_{0}, 1\right) \cdot[\delta F, \delta G]_{R_{k}}\right\rangle,
\end{aligned}
$$

where [,] here is the Virasoro Lie bracket, $[\phi \psi]=\left(\phi \psi^{\prime}-\phi^{\prime} \psi, \int \phi \psi^{\prime \prime \prime}\right) . \delta F$ is

$$
\lambda^{-1} \frac{\delta F}{\delta w_{0}}+\lambda^{-2} \frac{\delta F}{\delta w_{1}}+\lambda^{-3} \frac{\delta F}{\delta w_{2}}+\lambda^{-4} \frac{\delta F}{\delta w_{3}}
$$

and $\delta G$ is

$$
\lambda^{-1} \frac{\delta G}{\delta w_{0}}+\lambda^{-2} \frac{\delta G}{\delta w_{1}}+\lambda^{-3} \frac{\delta G}{\delta w_{2}}+\lambda^{-4} \frac{\delta G}{\delta w_{3}} .
$$

The five Poisson structures can be written - acting on $\left(\delta w_{0}, \delta w_{1}, \delta w_{2}, \delta w_{3}\right)$ - as

$$
\begin{array}{rlr}
K_{0} & =-\left(\begin{array}{cccc}
J_{1} & J_{2} & J_{3} & J_{4} \\
J_{2} & J_{3} & J_{4} & 0 \\
J_{3} & J_{4} & 0 & 0 \\
J_{4} & 0 & 0 & 0
\end{array}\right), \\
K_{2} & =\left(\begin{array}{cccc}
0 & J_{0} & 0 & 0 \\
J_{0} & J_{1} & 0 & 0 \\
0 & 0 & -J_{3} & -J_{4} \\
0 & 0 & -J_{4} & 0
\end{array}\right), & K_{1}=\left(\begin{array}{cccc}
J_{0} & 0 & 0 & 0 \\
0 & -J_{2} & -J_{3} & -J_{4} \\
0 & -J_{3} & -J_{4} & 0 \\
0 & -J_{4} & 0 & 0
\end{array}\right), \\
K_{4} & =\left(\begin{array}{cccc}
0 & 0 & 0 & J_{0} \\
0 & 0 & J_{0} & J_{1} \\
0 & J_{0} & J_{1} & J_{2} \\
J_{0} & J_{1} & J_{2} & J_{3}
\end{array}\right) .
\end{array}
$$

with

$$
\begin{aligned}
& J_{0}=\partial^{3}+w_{0} \partial+\partial w_{0}, \quad J_{1}=w_{1} \partial+\partial w_{1}, \\
& J_{2}=w_{2} \partial+\partial w_{2}, \quad J_{3}=w_{3} \partial+\partial w_{3}, \quad J_{4}=-2 \partial .
\end{aligned}
$$

It is straightforward to show that the push-forward map, $m_{*} B_{i}$, of $B_{i}$ induced by $m\left(u, \xi, e_{1}, e_{2}\right)=\left(e_{1} u-\frac{1}{2} \xi^{2}-e_{2} \xi^{\prime}, e_{2}{ }^{2}\right)$ is $K_{i+2}$ for $i=0,1,2$, i.e.

$$
m^{*}\left(\{f, g\}_{K_{\imath+2}}\right)\left(u, \xi, e_{1}, e_{2}\right)=\left(m^{*} f, m^{*} g\right\}_{B_{1}}\left(u, \xi, e_{1}, e_{2}\right)
$$


for any $f, g \in C^{\infty}\left(\tilde{\mathfrak{g}}^{*}\right)$, where $m^{*} f$ is given by

$$
m^{*} f\left(u, \xi, e_{1}, e_{2}\right)=f\left(e_{1} u-\frac{1}{2} \xi^{2}-e_{2} \xi^{\prime}, e_{2}^{2}\right) .
$$

One might like to see some flows:

Solving (23) recursively, we obtain the first two non-trivial Hamiltonians and their pull-backs,

$$
\begin{aligned}
& H_{0}(w)=\int 2 w_{3} \quad \text { and } \quad H_{1}(w)=\int\left(2 w_{2}+\frac{1}{2} w_{3}^{2}\right) ; \\
& F_{0}(u, \xi)=\int 2 u_{1} \quad \text { and } \quad F_{1}(u, \xi)=\int\left(2 u_{0}-\xi_{1}{ }^{2}+\frac{1}{2} u_{1}{ }^{2}\right) \text {. }
\end{aligned}
$$

With respect to the Hamiltonian structures given by $K_{4}$ and $B_{2}, H_{0}$ and $F_{0}$ give the translational flows, $w_{t_{0}}=w^{\prime}$ and $(u, \xi)_{t_{0}}=(u, \xi)^{\prime} ; H_{1}$ and $F_{1}$ give the flows

and

$$
\left(\begin{array}{l}
w_{0} \\
w_{1} \\
w_{2} \\
w_{3}
\end{array}\right)_{t_{1}}=\left(\begin{array}{l}
w_{3}^{\prime \prime \prime}+2 w_{0} w_{3}^{\prime}+w_{0}^{\prime} w_{3} \\
2 w_{0}^{\prime}+2 w_{1} w_{3}^{\prime}+w_{1}^{\prime} w_{3} \\
2 w_{1}^{\prime}+2 w_{2} w_{3}^{\prime}+w_{2}^{\prime} w_{3} \\
2 w_{2}^{\prime}+3 w_{3} w_{3}^{\prime}
\end{array}\right)
$$

$$
\left(\begin{array}{l}
u_{0} \\
\xi_{0} \\
u_{1} \\
\xi_{1}
\end{array}\right)_{t_{1}}=\left(\begin{array}{l}
-2 \xi_{0} \xi_{1}^{\prime}-2 \xi_{1}^{\prime \prime}+u_{0}{ }^{\prime} u_{1}+2 u_{0} u_{1}^{\prime} \\
\left(\xi_{0} u_{1}\right)^{\prime}-u_{1}^{\prime \prime} \\
2 u_{0}^{\prime}+3 u_{1} u_{1}^{\prime}-2 \xi_{1} \xi_{1}^{\prime} \\
2 \xi_{0}^{\prime}+\left(\xi_{1} u_{1}\right)^{\prime}
\end{array}\right) .
$$

\section{Corfespondence with a Spectral Problem}

Consider the spectral problem

$$
L \psi=\left(\begin{array}{cc}
\sigma & \varepsilon \partial+\eta \\
\varepsilon \partial-\eta & \kappa
\end{array}\right) \psi
$$

This spectral problem has the following origin:

The spectral problem (see [3])

$$
\left(\varepsilon^{2} \partial^{2}+\left(-v^{2}-v^{\prime}\right)+u_{1} \lambda+\cdots+u_{N-1} \lambda^{N-1}-\lambda^{N}\right) \phi=0
$$

is equivalent to

$$
\left((\varepsilon \partial+v)(\varepsilon \partial-v)+\lambda\left(u_{1}+\cdots+u_{N-1} \lambda^{N-2}-\lambda^{N-1}\right)\right) \phi=0 .
$$

If we set $\psi_{1}=\phi, \lambda \psi_{2}=(\varepsilon \partial-v) \phi$ we can write this as

$$
\begin{aligned}
(\varepsilon \partial+v) \psi_{2}+\left(u_{1}+\cdots+u_{N-1} \lambda^{N-2}-\lambda^{N-1}\right) \psi_{1} & =0 \\
(\varepsilon \partial-v) \psi_{1}-\lambda \psi_{2} & =0 .
\end{aligned}
$$

i.e.

$$
\left(\begin{array}{cc}
\left(u_{1}+\cdots+u_{N-2} \lambda^{N-2}-\lambda^{N-1}\right) & \varepsilon \partial+v \\
\varepsilon \partial-v & -\lambda
\end{array}\right)\left(\begin{array}{l}
\psi_{1} \\
\psi_{2}
\end{array}\right)=0
$$


Generalise this to

$$
L=\left(\begin{array}{cc}
\sigma & \varepsilon \partial+\eta \\
\varepsilon \partial-\eta & \kappa
\end{array}\right)
$$

where $\varepsilon, \kappa$, are constants.

The fact that any candidate compatible $\phi_{t}$ flow has the form

$$
\phi_{t}=\left(-\frac{1}{2} a^{\prime}+a \partial\right) \phi
$$

leads us to the ansatz

$$
\psi_{t}=V \psi
$$

with

$$
V=\left(\begin{array}{cc}
-\frac{1}{2} a^{\prime}+a \partial & 0 \\
x & y
\end{array}\right),
$$

where $x$ and $y$ are first order differential operators. We very easily find that $x$ and $y$ must have the form

$$
x=-\gamma^{\prime}, \quad y=\frac{1}{2} a^{\prime}+a \partial .
$$

The condition $\psi_{x t}=\psi_{t x}$ leads to $\left(L_{t}-[V, L]\right) \psi=0$. If we evaluate $[V, L]$ we find that we cannot have $L_{t}-[V, L]=0$, but that $\exists$ a matrix $A-A$ depends on $V$ - such that

$$
L_{t}-[V, L]=A L,
$$

and this of course is enough for $\left(L_{t}-[V, L]\right) \psi$ to vanish.

We next recognise that the set of matrix operators of the form

$$
\left(\begin{array}{cc}
-\frac{1}{2} a^{\prime}+a \partial & 0 \\
-\gamma^{\prime} & \frac{1}{2} a^{\prime}+a \partial
\end{array}\right)
$$

forms a Lie algebra under ordinary commutation of operators bracket. It seems reasonable therefore to postulate that the action of $V$ on $L$ given by

$$
V \circ L=[V, L]+A L
$$

is an $\mathrm{ad}^{*}$-action for some appropriate pairing of $(\sigma, \eta, \varepsilon, \kappa)$ with $(a, \gamma)$. The condition to be satisfied by this pairing is

$$
\left\langle\operatorname{ad}_{(a, \gamma)}^{*}(\sigma, \eta, \varepsilon, \kappa),(b . \delta)\right\rangle=-\langle(\sigma, \eta, \varepsilon, \kappa),[(a, \gamma)(b, \delta)]\rangle .
$$

If we go through these calculations we find that

$$
A=\left(\begin{array}{rr}
2 a^{\prime} & -\gamma^{\prime} \\
\gamma^{\prime} & 0
\end{array}\right)
$$

and

$$
\operatorname{ad}_{(a, \gamma)}^{*}(s, \eta, \varepsilon, \kappa)=\left(2 a^{\prime} \sigma+a \sigma^{\prime}+\varepsilon \gamma^{\prime \prime}+2 \eta \gamma^{\prime},(a \eta)^{\prime}-\frac{1}{2} \varepsilon a^{\prime \prime}-\kappa \gamma^{\prime}\right) .
$$

We also find that $[(a, \gamma)(b, \delta)]=\left(a b^{\prime}-a^{\prime} b, a \delta^{\prime}-b \gamma^{\prime}\right)$, and the pairing we require turns out to be

$$
\langle(\sigma, \eta, \varepsilon, \kappa),(a, \gamma, \mu, \nu)\rangle=\int \sigma a+2 \int \eta \gamma+\varepsilon \mu+\kappa \nu .
$$


( $\mu, v$ are just components put into account for a 2-dimensional extension which we realise is present in the process of doing this calculation) and

$$
[(a, \gamma)(b, \delta)]=\left(a b^{\prime}-a^{\prime} b, a \delta^{\prime}-b \gamma^{\prime},-\int\left(a^{\prime \prime} \delta-b^{\prime \prime} \gamma\right),-2 \int \delta \gamma^{\prime}\right) .
$$

This is remarkably like $\mathscr{L}, \mathscr{L}^{*}$, etc.!

In fact the only modification needed to transpose the analysis of the spectral problem in the coordinates given here to a suitable analysis in $\mathscr{L}$ and $\mathscr{L}^{*}$ is to adopt the inner product

$$
\langle(u, \xi),(\phi, v)\rangle=\int u \phi+2 \int \xi v
$$

in place of that given in Eq. (11), which will affect the computation of $\delta f$. This also changes the Miura map so that we set $w=e_{1} u-\xi^{2}-e_{2} \xi^{\prime}$; a slightly more standard form.

On the other hand we can easily adapt the spectral problem instead:

We use

$$
L \psi=\left(\begin{array}{cc}
2 u & 2 e_{2} \partial+\xi \\
2 e_{2} \partial-\xi & -e_{1}
\end{array}\right) \psi
$$

and use the same inner product used in the text.

We observe one further fact; we can adjust $L$ to

$$
L=\left(\begin{array}{cc}
2 u+4 e_{3} \partial^{2} & 2 e_{2} \partial+\xi \\
2 e_{2} \partial-\xi & -e_{1}
\end{array}\right)
$$

and (with the same $A$ )

$$
V \circ L=\left(\begin{array}{cc}
4 a^{\prime} u+2 a u^{\prime}+2 e_{2} \gamma^{\prime \prime}+2 \gamma^{\prime} \xi+2 e_{3} a^{\prime \prime \prime} & (a \xi)^{\prime}+e_{1} \gamma^{\prime}-e_{2} a^{\prime \prime} \\
-(a \xi)^{\prime}-e_{1} \gamma^{\prime}+e_{2} a^{\prime \prime} & 0
\end{array}\right)
$$

which is to be compared with Eq. (17). We thereby get a spectral problem representation of the integrable systems on $\mathscr{L}^{*}$.

There is a general remark to be made here: We often find that a linear problem

$$
\left\{\begin{array}{l}
L \psi=0 \\
V \psi=\psi_{t}
\end{array}\right.
$$

leads not to $L_{t}-[V, L]=0$, but to $L_{t}=[V, L]+A L=V \circ L$ say for some $A=A(V)$. The idea of looking for a pairing between $L$ and $V$ to make the action of $V$ on $L$ an ad*-action, i.e. such that $\operatorname{ad}^{*} L=V \circ L$, is always worth trying.

Note: Although $V=\left(\begin{array}{cc}a \partial & 0 \\ v & a \partial\end{array}\right)$ seems a more obvious way to represent $\mathscr{L}$, this does not give us a nice representation of $\tilde{\mathscr{L}}^{*}$.

\section{Comments}

There are some comments to be made about the cocycles $\sigma_{1}, \sigma_{2}, \sigma_{3}$. It seems that we have found a kind of factorisation of the Schwartzian derivative cocycle:

Notice that $\mathscr{G}$ can be written as the product of two subgroups

$$
\mathscr{G}=\mathscr{G}_{1} \times \mathscr{G}_{2}=\left\{\left(g, \log g^{\prime}\right)\right\} \times\{(e, v)\} .
$$


The Lie algebra decomposition corresponding to $\mathscr{G}=\mathscr{G}_{1} \times \mathscr{G}_{2}$ is $\mathscr{L}=\mathscr{L}_{1}+\mathscr{L}_{2}$ with $\mathscr{L}_{1}=\left\{\left(\phi, \phi^{\prime}\right)\right\}$ and $\mathscr{L}_{2}=\{(0, v)\}$. We find $\mathscr{L}^{*} \supset \mathscr{L}_{1}{ }^{\perp}=\left\{\left(\xi^{\prime}, \xi\right)\right\}$ and $\mathscr{L}^{*} \supset \mathscr{L}_{2}{ }^{\perp}=$ $\{(0, v)\}$. Hence $\mathscr{G}_{1}$ is a Lie group with Lie algebra $\mathscr{L}_{1}$ and dual $\mathscr{L}_{2}{ }^{\perp}$, while $\mathscr{G}_{2}$ is a Lie group with Lie algebra $\mathscr{L}_{2}$ and dual $\mathscr{L}_{1}{ }^{\perp}$.

Let $P_{1}, P_{2}$ be projections onto $\mathscr{L}_{1}$ and $\mathscr{L}_{2}$ respectively parallel to $\mathscr{L}_{2}$ and $\mathscr{L}_{1}$ respectively, and $\Pi_{1}, \Pi_{2}$ be projections with respect to $\mathscr{L}_{1}{ }^{\perp}$ and $\mathscr{L}_{2}{ }^{\perp}$ similarly.

Consider $\sigma_{1}\left(\phi, \phi^{\prime}\right)=\left(0, \phi^{\prime \prime}\right)=\left(\phi^{\prime \prime \prime}, \phi^{\prime}\right)+\left(-\phi^{\prime \prime \prime}, 0\right)$.

$\therefore \Pi_{2} \sigma_{1}\left(\phi, \phi^{\prime}\right)=\left(-\phi^{\prime \prime \prime}, 0\right)$.

Similarly $\Sigma_{1}\left(g, \log g^{\prime}\right)=\left(\frac{1}{2}\left(\frac{g^{\prime \prime}}{g^{\prime}}\right)^{2}, \frac{g^{\prime \prime}}{g^{\prime}}\right)=\left(\left(\frac{g^{\prime \prime}}{g^{\prime}}\right), \frac{g^{\prime \prime}}{g^{\prime}}\right)+\left(-\left(\frac{g^{\prime \prime}}{g^{\prime}}\right)^{2}+\frac{1}{2}\left(\frac{g^{\prime \prime}}{g^{\prime}}\right)^{2}, 0\right)$.

$\therefore \Pi_{2} \Sigma_{1}\left(g, \log g^{\prime}\right)=(-S(g), 0)$.

In the same way we find

$$
\begin{aligned}
\Pi_{2} \sigma_{2}\left(\phi, \phi^{\prime}\right) & =2\left(\phi^{\prime \prime \prime}, 0\right), \\
\Pi_{2} \Sigma_{2}\left(g, \log g^{\prime}\right) & =2(S(g), 0)
\end{aligned}
$$

and

$\Pi_{2} \sigma_{3}\left(\phi, \phi^{\prime}\right)=\Pi_{2}\left(\phi^{\prime \prime \prime}, 0\right)=\left(\phi^{\prime \prime \prime}, 0\right), \Pi_{2} \Sigma_{3}\left(g, \log g^{\prime}\right)=(S(g), 0)$.

Another remark is that $\mathscr{G}_{1} \cong \mathscr{G} / \mathscr{G}_{2}$, while in $\mathscr{L}^{*} / \mathscr{G}_{2}$.

$$
\left(u, \xi, e_{1}, e_{2}, e_{3}\right) \sim\left(u+v^{\prime} \xi+\frac{1}{2} e_{1} v^{\prime 2}+e_{2} v^{\prime \prime}, \xi+e_{1} v^{\prime}, e_{1}, e_{2}, e_{3}\right)
$$

for all $v \in \mathscr{V}$. In particular, choosing $e_{1} v^{\prime}=-\xi$, we get

$$
\left(u, \xi, e_{1}, e_{2}, e_{3}\right) \sim\left(u-\frac{\frac{1}{2}}{e_{1}} \underline{\xi}^{2}-\frac{e_{2}}{e_{1}} \xi^{\prime}, 0, e_{1}, e_{2}, e_{3}\right) \in \mathscr{L}_{2}{ }^{\perp} .
$$

I believe that these remarks allude to properties of $\mathscr{G}$ which I have not yet managed to uncover, but which may lead to a more through account of what is going on, cf. [7].

In conclusion I would like to remark that a complete Lie algebraic explanation of all the structures described in [3] is still lacking. This paper clearly points the way, and I hope eventually to do this job.

Acknowledgements. This work has been supported by a SERC Research Grant. Conversations with Andrew Crumey and Allan Fordy have useful.

\section{References}

1. Reyman, A. G., Semenov-Tian-Shansky, M. A.: Compatible poisson structure for Lax Equations: An $r$-matrix approach. Phys. Lett. 130, 456-460 (1988)

2. Fordy, A. P., Reyman, A. G., Semenov-Tian-Shansky, M. A.: Classical $r$-matrices and compatible poisson brackets for coupled kdV systems. Lett. Math. Phys. 17, 25-29 (1989)

3. Antonowicz, M, Fordy, A. P.: Factorisation of energy dependent Schrödinger operators: Miura maps and modified systems. Commun. Math. Phys. 124, 465-486 (1989)

4. Liberman, P., Marle, C.-M.: Symplectic geometry and analytical mechanics. Dordrecht: D. Reidel 1987

5. Kirillov, A. A.: Infinite dimensional Lie groups; their orbits, invariants and representations. 
The geometry of moments. Lecture Notes in Mathematics vol. 970. Berlin, Heidelberg, New York: Springer

6. Drinfeld, V. G.: Hamiltonian structures on Lie groups, Lie bialgebras and the geometrical meaning of the Yang-Baxter equations. Sov. Math. Doklady 27, 68 (1983)

7. Semenov-Tian-Shansky, M. A.: Dressing transformations and poisson group actions RIMS 21, 1237-1260 (1985)

8. Kupershmidt, B.: Mathematics of dispersive water waves. Commun. Math. Phys. 17, 51-73 (1985)

Communicated by $\mathrm{H}$. Araki 